International Journal of Pure and Applied Mathematics

Volume 114 No. $4 \quad 2017,881-893$

ISSN: 1311-8080 (printed version); ISSN: 1314-3395 (on-line version)

url: http://www.ijpam.eu

doi: 10.12732/ijpam.v114i4.18

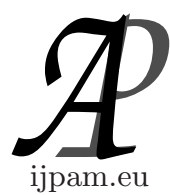

\title{
$L$-FUZZY $(K, E)$-SOFT TOPOLOGIES AND $L$-FUZZY $(K, E)$-SOFT CLOSURE OPERATORS INDUCED BY FUZZY SOFT MAPS
}

\author{
Jin Won Park ${ }^{1}$, Yong Chan Kim² ${ }^{2}$ \\ ${ }^{1}$ Department of Mathematics Education \\ Jeju, 63243, National University \\ Jeju, KOREA \\ ${ }^{2}$ Department of Mathematics \\ Gangneung-Wonju University \\ Gangneung, Gangwondo, 25558, KOREA
}

\begin{abstract}
In this paper, we study $L$-fuzzy $(K, E)$-soft topologies and $L$-fuzzy $(K, E)$-soft closure spaces induced by fuzzy soft maps in complete residuated lattices. We give some examples.
\end{abstract}

AMS Subject Classification: 03E72, 06A15, 06F07, 54A05

Key Words: complete residuated lattices, fuzzy soft sets, fuzzy soft map, $L$-fuzzy $(K, E)$ soft topologies, $L$-fuzzy $(K, E)$-soft closure operators

\section{Introduction}

In 1999, Molodtsov [13] initiated the theory of soft sets as a new mathematical tool to deal with uncertainties while modeling problems in engineering physics, computer science, economics, social sciences and medical sciences. In [14], Molodtsov applied successfully in directions such as, smoothness of functions, game theory, operations research, Riemann-integration, Perron integra-

Received: $\quad$ March 14, 2017

Revised: $\quad$ May 10, 2017

Published: June 7, 2017

(c) 2017 Academic Publications, Ltd.

url: www.acadpubl.eu

${ }^{\S}$ Correspondence author 
tion, probability and theory of measurement. Maji et al. [10,11] gave the first practical application of soft sets in decision making problems. In 2003, Maji et al. [11] defined and studied several basic notions of soft set theory. Many researchers have contributed towards the algebraic structure of soft set theory [1-5,7]. In 2011, Shabir and Naz [19] initiated the study of soft topological spaces. They defined soft topology on the collection of soft sets over $\mathrm{X}$ and established their several properties. Aygünoglu et.al [2] introduced the concept of $(K, E)$-soft topology in the sense of Šostak [9]. Cetkin et.al [3] studied $(K, E)$-soft proximities and discuss their properties.

Hájek [8] introduced a complete residuated lattice which is an algebraic structure for many valued logic and decision rules in complete residuated lattices. Höhle [9] introduced $L$-fuzzy topologies with algebraic structure $L$ (cqm, quantales, $M V$-algebra). It has developed in many directions $[16,17]$. Kim et al. [10] defined $L$-fuzzy $(K, E)$-soft topologies and $L$-fuzzy $(K, E)$-soft closure spaces in complete residuated lattices.

In this paper, we study $L$-fuzzy $(K, E)$-soft topologies and $L$-fuzzy $(K, E)$ soft closure spaces induced by fuzzy soft maps in complete residuated lattices. We give their examples.

\section{Preliminaries}

Definition 1. $[8,9]$ An algebra $(L, \wedge, \vee, \odot, \rightarrow, 0,1)$ is called a complete residuated lattice if it satisfies the following conditions:

(C1) $L=(L, \leq, \vee, \wedge, 1,0)$ is a complete lattice with the greatest element 1 and the least element 0 ;

(C2) $(L, \odot, 1)$ is a commutative monoid;

(C3) $x \odot y \leq z$ iff $x \leq y \rightarrow z$ for $x, y, z \in L$.

In this paper, we always assume that $\left(L, \leq, \odot, \rightarrow, \oplus,^{*}\right)$ is a complete residuated lattice with an order reversing involution * which is defined by

$$
x \oplus y=\left(x^{*} \odot y^{*}\right)^{*}, \quad x^{*}=x \rightarrow 0
$$

unless otherwise specified.

Remark 2. Every completely distributive lattice $\left(L, \leq, \odot=\wedge, \oplus=\vee{ }^{*}\right)$ with order reversing involution * is a complete residuated lattice.

Lemma 3. $[8,9]$ For each $x, y, z, x_{i}, y_{i}, w \in L$, we have the following properties.

(1) $1 \rightarrow x=x, 0 \odot x=0$, 
(2) If $y \leq z$, then $x \odot y \leq x \odot z, x \oplus y \leq x \oplus z, x \rightarrow y \leq x \rightarrow z$ and $z \rightarrow x \leq y \rightarrow x$,

(3) $x \leq y$ iff $x \rightarrow y=1$.

(4) $\left(\bigwedge_{i} y_{i}\right)^{*}=\bigvee_{i} y_{i}^{*},\left(\bigvee_{i} y_{i}\right)^{*}=\bigwedge_{i} y_{i}^{*}$,

(5) $x \rightarrow\left(\bigwedge_{i} y_{i}\right)=\bigwedge_{i}\left(x \rightarrow y_{i}\right)$,

(6) $\left(\bigvee_{i} x_{i}\right) \rightarrow y=\bigwedge_{i}\left(x_{i} \rightarrow y\right)$,

(7) $x \rightarrow\left(\bigvee_{i} y_{i}\right) \geq \bigvee_{i}\left(x \rightarrow y_{i}\right)$,

(8) $\left(\bigwedge_{i} x_{i}\right) \rightarrow y \geq \bigvee_{i}\left(x_{i} \rightarrow y\right)$,

(9) $(x \odot y) \rightarrow z=x \rightarrow(y \rightarrow z)=y \rightarrow(x \rightarrow z)$,

(10) $x \odot y=\left(x \rightarrow y^{*}\right)^{*}$ and $x \oplus y=x^{*} \rightarrow y$,

(11) $(x \rightarrow y) \odot(z \rightarrow w) \leq(x \odot z) \rightarrow(y \odot w)$,

(12) $x \rightarrow y \leq(x \odot z) \rightarrow(y \odot z)$ and $(x \rightarrow y) \odot(y \rightarrow z) \leq x \rightarrow z$,

(13) $(x \rightarrow y) \odot(z \rightarrow w) \leq(x \oplus z) \rightarrow(y \oplus w)$.

(14) $x \rightarrow y=y^{*} \rightarrow x^{*}$.

(15) $(x \vee y) \odot(z \vee w) \leq(x \vee z) \vee(y \odot w) \leq(x \oplus z) \vee(y \odot w)$. $\left.y_{i}\right)$,

(16) $\bigvee_{i \in \Gamma} x_{i} \rightarrow \bigvee_{i \in \Gamma} y_{i} \geq \bigwedge_{i \in \Gamma}\left(x_{i} \rightarrow y_{i}\right)$ and $\bigwedge_{i \in \Gamma} x_{i} \rightarrow \bigwedge_{i \in \Gamma} y_{i} \geq \bigwedge_{i \in \Gamma}\left(x_{i} \rightarrow\right.$

(17) $(x \odot y) \odot(z \oplus w) \leq(x \odot z) \oplus(y \odot w)$.

Throughout this paper, $X$ refers to an initial universe, $E$ and $K$ are the sets of all parameters for $X$, and $L^{X}$ is the set of all $L$-fuzzy sets on $X$.

Definition 4. [4] A map $f$ is called an $L$-fuzzy soft set on $X$, where $f$ is a mapping from $E$ into $L^{X}$, i.e., $f_{e}:=f(e)$ is an $L$ - fuzzy set on $X$, for each $e \in E$. The family of all $L$ - fuzzy soft sets on $X$ is denoted by $\left(L^{X}\right)^{E}$. Let $f$ and $g$ be two $L$ - fuzzy soft sets on $X$.

(1) $f$ is an $L$-fuzzy soft subset of $g$ and we write $f \sqsubseteq g$ if $f_{e} \leq g_{e}$, for each $e \in E$. $f$ and $g$ are equal if $f \sqsubseteq g$ and $g \sqsubseteq f$.

(2) The intersection of $f$ and $g$ is an $L$ - fuzzy soft set $h=f \sqcap g$, where $h_{e}=f_{e} \wedge g_{e}$, for each $e \in E$.

(3) The union of $f$ and $g$ is an $L$ - fuzzy soft set $h=f \sqcup g$, where $h_{e}=f_{e} \vee g_{e}$, for each $e \in E$.

(4) An $L$ - fuzzy soft set $h=f \odot g$ is defined as $h_{e}=f_{e} \odot g_{e}$, for each $e \in E$.

(5) An $L$ - fuzzy soft set $h=f \oplus g$ is defined as $h_{e}=f_{e} \oplus g_{e}$, for each $e \in E$.

(6) The complement of an $L$ - fuzzy soft sets on $X$ is denoted by $f^{*}$, where $f^{*}: E \rightarrow L^{X}$ is a mapping given by $f_{e}^{*}=\left(f_{e}\right)^{*}$, for each $e \in E$.

(7) $f$ is called a null $L$ - fuzzy soft set and is denoted by $0_{X}$, if $f_{e}(x)=0$, for each $e \in E, x \in X$.

(8) $f$ is called an absolute $L$ - fuzzy soft set and is denoted by $1_{X}$, if $f_{e}(x)=1$, for each $e \in E, x \in X$ and $\left(1_{X}\right)_{e}(x)=1$. 
Definition 5. [2,4] Let $\varphi: X \rightarrow Y$ and $\psi: E_{1} \rightarrow E_{2}$ be two mappings, where $E_{1}$ and $E_{2}$ are parameters sets for the crisp sets $X$ and $Y$, respectively. Then $\varphi_{\psi}:\left(L^{X}\right)^{E_{1}} \rightarrow\left(L^{Y}\right)^{E_{2}}$ is called a fuzzy soft mapping.

(1) For $f \in\left(L^{X}\right)^{E_{1}}$, the image of $f$ under the fuzzy soft mapping $\varphi_{\psi}$ defined by, $\forall k \in K, \forall y \in Y$,

$$
\varphi(f)_{e_{2}}(y)= \begin{cases}\bigvee_{\varphi(x)=y}\left(\bigvee_{\psi\left(e_{1}\right)=e_{2}} f_{e_{1}}(x)\right), & \text { if } x \in \varphi^{-1}(y), e_{1} \in \psi^{-1}\left(e_{2}\right) \\ 0, & \text { otherwise. }\end{cases}
$$

(2) For $f \in\left(L^{X}\right)^{E_{1}}$, the pre-image of $g$ defined by

$$
\varphi_{\psi}^{-1}(g)_{e}(x)=g_{\psi(e)}(\varphi(x)), \forall e \in E, \forall x \in X
$$

(3) The fuzzy soft mapping $\varphi_{\psi}:\left(L^{X}\right)^{E_{1}} \rightarrow\left(L^{Y}\right)^{E_{2}}$ is called injective (resp. surjective, bijective) if $f$ and $\phi$ are both injective (resp. surjective, bijective).

Definition 6. $[2,10]$ A mapping $\mathcal{T}: K \rightarrow L^{\left(L^{X}\right)^{E}}$ (where $\mathcal{T}_{k}:=\mathcal{T}(k)$ : $\left(L^{X}\right)^{E} \rightarrow L$ is a mapping for each $\left.k \in K\right)$ is called an $L$-fuzzy $(K, E)$-soft topology on $X$ if it satisfies the following conditions for each $k \in K$.

(O1) $\mathcal{T}_{k}\left(0_{X}\right)=\mathcal{T}_{k}\left(1_{X}\right)=1$,

(O2) $\mathcal{T}_{k}(f \odot g) \geq \mathcal{T}_{k}(f) \odot \mathcal{T}_{k}(g) \forall f, g \in\left(L^{X}\right)^{E}$,

(O3) $\mathcal{T}_{k}\left(\bigsqcup_{i} f_{i}\right) \geq \bigwedge_{i \in I} \mathcal{T}_{k}\left(f_{i}\right) \forall f_{i} \in\left(L^{X}\right)^{E}, i \in I$.

The pair $(X, \mathcal{T})$ is called an $L$-fuzzy $(K, E)$-soft topological space. Let $\mathcal{T}^{1}$ and $\mathcal{T}^{2}$ be $L$-fuzzy $(K, E)$-soft topologies on $X$. Then $\mathcal{T}^{1}$ is coarser than $\mathcal{T}^{2}$ if $\mathcal{T}_{k}^{1}(f) \leq \mathcal{T}_{k}^{2}(f)$, for all $f \in\left(L^{X}\right)^{E}, k \in K$.

Let $\left(X, \mathcal{T}^{1}\right)$ be an $L$-fuzzy $\left(K_{1}, E_{1}\right)$-soft topological space and $\left(Y, \mathcal{T}^{2}\right)$ be an $L$-fuzzy $\left(K_{2}, E_{2}\right)$-soft topological space. Let $\varphi: X \rightarrow Y, \psi: E_{1} \rightarrow E_{2}$ and $\eta: K_{1} \rightarrow K_{2}$ be mappings. Then $\varphi_{\psi, \eta}$ from $\left(X, \mathcal{T}^{1}\right)$ into $\left(Y, \mathcal{T}^{2}\right)$ is called $L$-fuzzy soft continuous if

$$
\mathcal{T}_{\eta(k)}^{2}(f) \leq \mathcal{T}_{k}^{1}\left(\varphi_{\psi}^{-1}(f)\right) \quad \forall f \in\left(L^{Y}\right)^{E_{2}}, k \in K_{1}
$$

Definition 7. [10] A map $\mathcal{C}: K \times\left(L^{X}\right)^{E} \times L_{0} \rightarrow\left(L^{X}\right)^{E}$ is called an $L$ fuzzy $(K, E)$-soft closure operator if it satisfies the following conditions;

(C1) $\mathcal{C}\left(k, 0_{X}, r\right)=0_{X}$,

(C2) $\mathcal{C}(k, f, r) \sqsupseteq f$,

(C3) If $f_{1} \sqsubseteq f_{2}$, then $\left.\mathcal{C}\left(k, f_{1}, r\right) \sqsubseteq \mathcal{C}\left(k, f_{2}, r\right)\right)$,

(C4) If $r_{1} \leq r_{2}$, then $\mathcal{C}\left(k, f, r_{1}\right) \sqsubseteq \mathcal{C}\left(k, f, r_{2}\right)$,

(C5) $\mathcal{C}\left(k, f_{1} \oplus f_{2}, r \odot s\right) \sqsubseteq \mathcal{C}\left(k, f_{1}, r\right) \oplus \mathcal{C}\left(k, f_{2}, s\right)$.

The pair $(X, \mathcal{C})$ is called an $L$-fuzzy $(K, E)$-soft closure space. 
An $L$ - fuzzy $(K, E)$-soft closure operator is called topological if (T) $\mathcal{C}(k, \mathcal{C}(k, f, r), r) \sqsubseteq \mathcal{C}(k, f, r)$.

Let $\mathcal{C}_{1}$ and $\mathcal{C}_{2}$ be $L$-fuzzy $(K, E)$-soft closure operators on $X$. Then $\mathcal{C}_{1}$ is finer than $\mathcal{C}_{2}$ if $\mathcal{C}_{1}(k, f, r) \sqsubseteq \mathcal{C}_{2}(k, f, r)$, for all $f \in\left(L^{X}\right)^{E}, r \in L_{0}$.

Let $\left(X, \mathcal{C}_{X}\right)$ be $L$-fuzzy $\left(K_{1}, E_{1}\right)$-soft closure spaces and $\left(Y, \mathcal{C}_{Y}\right)$ be $L$-fuzzy $\left(K_{2}, E_{2}\right)$-soft closure spaces. Let $\varphi: X \rightarrow Y, \psi: E_{1} \rightarrow E_{2}$ and $\eta: K_{1} \rightarrow K_{2}$ be maps. Then $\varphi_{\psi, \eta}$ is called an $L$-fuzzy soft closed map if, for each $k \in K_{1}, f \in$ $\left(L^{X}\right)^{E_{1}}, r \in L_{0}$,

$$
\varphi_{\psi, \eta}\left(\mathcal{C}_{X}(k, f, r)\right) \sqsubseteq \mathcal{C}_{Y}\left(\eta(k), \varphi_{\psi}(f), r\right) .
$$

Lemma 8. [10] Let $\varphi_{\psi}:\left(L^{X}\right)^{E_{1}} \rightarrow\left(L^{Y}\right)^{E_{2}}$ be a fuzzy soft mapping. Then we have the following properties. For $f, f_{i} \in\left(L^{X}\right)^{E_{1}}$ and $g, g_{i} \in\left(L^{Y}\right)^{E_{2}}$,

(1) $g \sqsupseteq \varphi_{\psi}\left(\varphi_{\psi}^{-1}(g)\right)$ with equality if $\varphi_{\psi}$ is surjective,

(2) $f \sqsubseteq \varphi_{\psi}^{-1}\left(\varphi_{\psi}(f)\right)$ with equality if $\varphi_{\psi}$ is injective,

(3) if $\varphi_{\psi}$ is injective,

$$
\varphi(f)_{e_{2}}(y)= \begin{cases}f_{e_{1}}(x), & \text { if } x \in \varphi^{-1}(\{y\}), e_{1} \in \psi^{-1}\left(\left\{e_{2}\right\}\right) \\ 0, & \text { otherwise, }\end{cases}
$$

(4) $\varphi_{\psi}^{-1}\left(g^{*}\right)=\left(\varphi_{\psi}^{-1}(g)\right)^{*}$,

(5) $\varphi_{\psi}^{-1}\left(\bigvee_{i \in I} g_{i}\right)=\bigvee_{i \in I} \varphi_{\psi}^{-1}\left(g_{i}\right)$,

(6) $\varphi_{\psi}^{-1}\left(\bigwedge_{i \in I} g_{i}\right)=\bigwedge_{i \in I} \varphi_{\psi}^{-1}\left(g_{i}\right)$,

(7) $\varphi_{\psi}\left(\bigvee_{i \in I} f_{i}\right)=\bigvee_{i \in I} \varphi_{\psi}\left(f_{i}\right)$

(8) $\varphi_{\psi}\left(\bigwedge_{i \in I} f_{i}\right) \sqsubseteq \bigwedge_{i \in I} \varphi_{\psi}\left(f_{i}\right)$ with equality if $\varphi_{\psi}$ is injective,

(9) $\varphi_{\psi}^{-1}\left(g_{1} \odot g_{2}\right)=\varphi_{\psi}^{-1}\left(g_{1}\right) \odot \varphi_{\psi}^{-1}\left(g_{2}\right)$,

(10) $\varphi_{\psi}^{-1}\left(g_{1} \oplus g_{2}\right)=\varphi_{\psi}^{-1}\left(g_{1}\right) \oplus \varphi_{\psi}^{-1}\left(g_{2}\right)$,

(11) $\varphi_{\psi}\left(f_{1} \odot f_{2}\right) \sqsubseteq \varphi_{\psi}\left(f_{1}\right) \odot \varphi_{\psi}\left(f_{2}\right)$ with equality if $\varphi_{\psi}$ is injective,

(12) $\varphi_{\psi}\left(f_{1} \oplus f_{2}\right) \sqsubseteq \varphi_{\psi}\left(f_{1}\right) \oplus \varphi_{\psi}\left(f_{2}\right)$ with equality if $\varphi_{\psi}$ is injective.

Theorem 9. [10] (1) Let $(X, \mathcal{T})$ be an $L$-fuzzy $(K, E)$-soft topological space. Define $\mathcal{C}_{\mathcal{T}}: K \times\left(L^{X}\right)^{E} \times L_{0} \rightarrow\left(L^{X}\right)^{E}$ as

$$
\mathcal{C}_{\mathcal{T}}(k, f, r)=\bigwedge\left\{g \in\left(L^{X}\right)^{E} \mid g \sqsupseteq f, \mathcal{T}_{k}\left(g^{*}\right) \geq r\right\} .
$$

Then $(1) \mathcal{C}_{\mathcal{T}}$ is a topological $L$-fuzzy $(K, E)$-soft closure operator.

(2) Let $(X, \mathcal{C})$ be an $L$-fuzzy $(K, E)$-soft closure space. Define $\mathcal{T}_{\mathcal{C}}:\left(L^{X}\right)^{E} \rightarrow$ $L$ as

$$
\left(\mathcal{T}_{\mathcal{C}}\right)_{k}(f)=\bigvee\left\{r \in L \mid \mathcal{C}\left(k, f^{*}, r\right) \sqsubseteq f^{*}\right\} .
$$

Then $\mathcal{T}_{\mathcal{C}}$ is an $L$-fuzzy $(K, E)$-soft topology on $X$.

(3) Let $(X, \mathcal{T})$ be an $L$-fuzzy $(K, E)$-soft topological space. Then $\mathcal{T}=\mathcal{T}_{\mathcal{C}_{\mathcal{T}}}$. 
Theorem 10. [10] Let $\left(X, \mathcal{T}_{X}\right)$ and $\left(Y, \mathcal{T}_{Y}\right)$ be $L$-fuzzy $\left(K_{1}, E_{1}\right)$-soft and $L$ fuzzy $\left(K_{2}, E_{2}\right)$-soft topological spaces, respectively. Let $\varphi_{\psi}:\left(L^{X}\right)^{E_{1}} \rightarrow\left(L^{Y}\right)^{E_{2}}$ be a soft map. If $\varphi_{\psi, \eta}:\left(X, \mathcal{T}_{X}\right) \rightarrow\left(Y, \mathcal{T}_{Y}\right)$ is an $L$-fuzzy soft continuous map, then $\varphi_{\psi, \eta}:\left(X, \mathcal{C}_{\mathcal{T}_{X}}\right) \rightarrow\left(Y, \mathcal{C}_{\mathcal{T}_{Y}}\right)$ is an $L$-fuzzy soft closure map.

Theorem 11. [10] Let $\left(X, \mathcal{C}_{X}\right)$ and $\left(Y, \mathcal{C}_{Y}\right)$ be $L$-fuzzy $\left(K_{1}, E_{1}\right)$-soft and $L$-fuzzy $\left(K_{2}, E_{2}\right)$-soft closure spaces, respectively. Let $\varphi_{\psi}:\left(L^{X}\right)^{E_{1}} \rightarrow\left(L^{Y}\right)^{E_{2}}$ be a fuzzy soft map. Then $\varphi_{\psi, \eta}:\left(X, \mathcal{C}_{X}\right) \rightarrow\left(Y, \mathcal{C}_{Y}\right)$ is an $L$-fuzzy soft closure map iff $\mathcal{C}_{X}\left(k, \varphi_{\psi}^{-1}(g), r\right) \sqsubseteq \varphi_{\psi}^{-1}\left(\mathcal{C}_{Y}(\eta(k), g, r)\right)$.

Theorem 12. [10] Let $\left(X, \mathcal{C}_{X}\right)$ and $\left(Y, \mathcal{C}_{Y}\right)$ be $L$-fuzzy $\left(K_{1}, E_{1}\right)$-soft and $L$-fuzzy $\left(K_{2}, E_{2}\right)$-soft closure spaces, respectively. Let $\varphi_{\psi}:\left(L^{X}\right)^{E} \rightarrow\left(L^{Y}\right)^{E_{2}}$ be a fuzzy soft map. Then the following properties;

(1) If $\varphi_{\psi, \eta}:\left(X, \mathcal{C}_{X}\right) \rightarrow\left(Y, \mathcal{C}_{Y}\right)$ is an $L$-fuzzy soft closure map, then $\varphi_{\psi, \eta}:$ $\left(X, \mathcal{T}_{\mathcal{C}_{X}}\right) \rightarrow\left(Y, \mathcal{T}_{\mathcal{C}_{Y}}\right)$ is an $L$-fuzzy soft continuous map.

(2) $\varphi_{\psi, \eta}:\left(X, \mathcal{T}_{X}\right) \rightarrow\left(Y, \mathcal{T}_{Y}\right)$ is an $L$-fuzzy soft continuous map iff $\varphi_{\psi, \eta}:$ $\left(X, \mathcal{C}_{\mathcal{T}_{X}}\right) \rightarrow\left(Y, \mathcal{C}_{\mathcal{T}_{Y}}\right)$ is an $L$-fuzzy soft closure map.

\section{3. $L$-fuzzy $(K, E)$-soft topologies and $L$-fuzzy $(K, E)$-soft closure operators induced by fuzzy soft maps}

Theorem 13. Let $\left(Y, \mathcal{T}_{Y}\right)$ be $L$-fuzzy $(K, E)$-soft topological spaces and $\varphi_{\psi}:\left(L^{X}\right)^{E} \rightarrow\left(L^{Y}\right)^{E_{2}}$ be a soft map. Define $\mathcal{T}_{X}: K \rightarrow L^{\left(L^{X}\right)^{E}}$ as

$$
\left(\mathcal{T}_{X}\right)_{k}(f)=\bigvee\left\{\left(\mathcal{T}_{Y}\right)_{\eta(k)}(g) \mid f=\varphi_{\psi}^{-1}(g)\right\}
$$

Then $\mathcal{T}_{X}$ is the coarsest $L$-fuzzy $(K, E)$-soft topology for which $\varphi_{\psi, \eta}:\left(X, \mathcal{T}_{X}\right) \rightarrow$ $\left(Y, \mathcal{T}_{Y}\right)$ is an $L$-fuzzy soft continuous map.

Proof. (O1) is easily proved from the definition of $\mathcal{T}_{X}$.

$$
\begin{aligned}
& \left(\mathcal{T}_{X}\right)_{k}\left(f_{1}\right) \odot\left(\mathcal{T}_{X}\right)_{k}\left(f_{2}\right) \\
& =\left(\bigvee\left\{\left(\mathcal{T}_{Y}\right)_{\eta(k)}\left(g_{1}\right) \mid f_{1}=\varphi_{\psi}^{-1}\left(g_{1}\right)\right\}\right) \odot \bigvee\left\{\left(\mathcal{T}_{Y}\right)_{\eta(k)}\left(g_{2}\right) \mid f_{2}=\varphi_{\psi}^{-1}\left(g_{2}\right)\right\} \\
& =\bigvee\left\{\left(\mathcal{T}_{Y}\right)_{\eta(k)}\left(g_{1}\right) \odot\left(\mathcal{T}_{Y}\right)_{\eta(k)}\left(g_{2}\right) \mid f_{1} \odot f_{2}=\varphi_{\psi}^{-1}\left(g_{1}\right) \odot \varphi_{\psi}^{-1}\left(g_{2}\right)\right\} \\
& \leq \bigvee\left\{\left(\mathcal{T}_{Y}\right)_{\eta(k)}\left(g_{1} \odot g_{2}\right) \mid f_{1} \odot f_{2}=\varphi_{\psi}^{-1}\left(g_{1} \odot g_{2}\right)\right\} \\
& =\left(\mathcal{T}_{X}\right)_{k}\left(f_{1} \odot f_{2}\right) .
\end{aligned}
$$


(O3)

$$
\begin{aligned}
\bigwedge_{i \in \Gamma}\left(\mathcal{T}_{X}\right)_{k}\left(f_{i}\right) & =\bigwedge_{i \in \Gamma}\left(\bigvee\left\{\left(\mathcal{T}_{Y}\right)_{\eta(k)}\left(g_{i}\right) \mid f_{i}=\varphi_{\psi}^{-1}\left(g_{i}\right)\right\}\right) \\
& =\bigvee\left\{\bigwedge_{i \in \Gamma}\left(\mathcal{T}_{Y}\right)_{\eta(k)}\left(g_{i}\right) \mid \bigvee_{i \in \Gamma} f_{i}=\varphi_{\psi}^{-1}\left(\bigvee_{i \in \Gamma} g_{i}\right)\right\} \\
& =\left(\mathcal{T}_{X}\right)_{k}\left(\bigvee_{i \in \Gamma} f_{i}\right)
\end{aligned}
$$

Let $\varphi_{\psi, \eta}:(X, \mathcal{T}) \rightarrow\left(Y, \mathcal{T}_{Y}\right)$ be an $L$-fuzzy soft continuous map. Then $\mathcal{T}_{k}\left(\varphi_{\psi}^{-1}(g)\right) \geq\left(\mathcal{T}_{Y}\right)_{\eta(k)}(g)$. Hence

$$
\mathcal{T}_{k}\left(\varphi_{\psi}^{-1}(g)\right) \geq\left(\mathcal{T}_{X}\right)_{k}\left(\varphi_{\psi}^{-1}(g)\right)
$$

Thus $\mathcal{T}_{k} \geq\left(\mathcal{T}_{X}\right)_{k}$

Theorem 14. Let $\left(Y, \mathcal{C}_{Y}\right)$ be a (resp. topological) $L$-fuzzy $\left(K_{2}, E_{2}\right)$-soft closure space, $\varphi_{\psi}:\left(L^{X}\right)^{E_{1}} \rightarrow\left(L^{Y}\right)^{E_{2}}$ be a soft map and $\eta: E_{1} \rightarrow E_{2}$. Define $\mathcal{C}_{X}: K_{1} \times\left(L^{X}\right)^{E_{1}} \times L_{0} \rightarrow\left(L^{X}\right)^{E_{1}}$ as

$$
\mathcal{C}_{X}(k, f, r)=\varphi_{\psi, \eta}^{-1}\left(\mathcal{C}_{Y}\left(\eta(k), \varphi_{\psi}(f), r\right)\right)
$$

Then $\mathcal{C}_{X}$ is the coarsest (resp. topological) $L$-fuzzy $\left(K_{1}, E_{1}\right)$-soft closure operator for which $\varphi_{\psi, \eta}:\left(X, \mathcal{C}_{X}\right) \rightarrow\left(Y, \mathcal{C}_{Y}\right)$ is an $L$-fuzzy soft closure map.

Proof. (C1)-(C3) and (C4) are easily proved from the definition of $\mathcal{C}_{X}$.

$$
\begin{aligned}
& \mathcal{C}_{X}\left(k, f_{1}, r\right) \oplus \mathcal{C}_{X}\left(k, f_{2}, s\right) \\
& =\varphi_{\psi, \eta}^{-1}\left(\mathcal{C}_{Y}\left(\eta(k), \varphi_{\psi}\left(f_{1}\right), r\right)\right) \oplus \varphi_{\psi, \eta}^{-1}\left(\mathcal{C}_{Y}\left(\eta(k), \varphi_{\psi}\left(f_{2}\right), s\right)\right) \\
& =\varphi_{\psi, \eta}^{-1}\left(\mathcal{C}_{Y}\left(\eta(k), \varphi_{\psi}\left(f_{1}\right), r\right) \oplus \mathcal{C}_{Y}\left(\eta(k), \varphi_{\psi}\left(f_{2}\right), s\right)\right) \\
& \sqsupseteq \varphi_{\psi, \eta}^{-1}\left(\mathcal{C}_{Y}\left(\eta(k), \varphi_{\psi}\left(f_{1}\right) \oplus \varphi_{\psi}\left(f_{2}\right), r \odot s\right)\right) \\
& \sqsupseteq \varphi_{\psi, \eta}^{-1}\left(\mathcal{C}_{Y}\left(\eta(k), \varphi_{\psi}\left(f_{1} \oplus f_{2}\right), r \odot s\right)\right) \\
& =\mathcal{C}_{X}\left(k, f_{1} \odot f_{2}, r \odot s\right) .
\end{aligned}
$$

From the definition of $\mathcal{C}_{X}$,

$$
\begin{aligned}
\varphi_{\psi, \eta}\left(\mathcal{C}_{X}(k, f, r)\right) & =\varphi_{\psi, \eta}\left(\varphi_{\psi, \eta}^{-1}\left(\mathcal{C}_{Y}\left(\eta(k), \varphi_{\psi, \eta}(f), r\right)\right)\right) \\
& \left.\sqsubseteq \mathcal{C}_{Y}\left(\eta(k), \varphi_{\psi, \eta}(f), r\right)\right) .
\end{aligned}
$$

Hence $\varphi_{\psi, \eta}:\left(X, \mathcal{C}_{X}\right) \rightarrow\left(Y, \mathcal{C}_{Y}\right)$ is an $L$-fuzzy soft closure map.

If $\varphi_{\psi, \eta}:(X, \mathcal{C}) \rightarrow\left(Y, \mathcal{C}_{Y}\right)$ is an $L$-fuzzy soft closure map, then $\varphi_{\psi, \eta}(\mathcal{C}(k, f, r)) \sqsubseteq$ $\mathcal{C}_{Y}\left(\eta(k), \varphi_{\psi}(f), r\right)$. Thus $\mathcal{C}(k, f, r) \sqsubseteq \varphi_{\psi}^{-1}\left(\mathcal{C}_{Y}\left(\eta(k), \varphi_{\psi}(f), r\right)\right)=\mathcal{C}_{X}(k, f, r)$. 
If $\mathcal{C}_{Y}$ is topological, then

$$
\begin{aligned}
& \mathcal{C}_{X}\left(k, \mathcal{C}_{X}(k, f, r), r\right) \\
& =\varphi_{\psi, \eta}^{-1}\left(\mathcal{C}_{Y}\left(\eta(k), \varphi_{\psi}\left(\mathcal{C}_{X}(k, f, r)\right), r\right)\right) \\
& \sqsubseteq \varphi_{\psi}^{-1}\left(\mathcal{C}_{Y}\left(\eta(k), \mathcal{C}_{Y}\left(\eta(k), \varphi_{\psi}(f), r\right)\right), r\right) \\
& =\varphi_{\psi}^{-1}\left(\mathcal{C}_{Y}\left(\eta(k), \varphi_{\psi}(f), r\right)\right)=\mathcal{C}_{X}(k, f, r) .
\end{aligned}
$$

Hence $\mathcal{C}_{X}$ is topological.

Example 15. Let $X=\left\{h_{i} \mid i=\{1, \ldots, 5\}\right\}$ with $h_{i}=$ house and and $E_{X}=$ $\{e, b, w, c\}$ with $e=$ expensive, $b=$ beautiful, $w=$ wooden, $c=$ creative. Define a binary operation $\wedge$ on $[0,1]$ by

$$
\begin{gathered}
x \wedge y=\min \{x, y\}, x^{*}=1-x, x \vee y=\left(x^{*} \wedge y^{*}\right)^{*} \\
x \rightarrow y= \begin{cases}1, & \text { if } x \leq y, \\
y, & \text { otherwise } .\end{cases}
\end{gathered}
$$

Then $([0,1], \wedge, \rightarrow, 0,1)$ is a complete residuated lattice (ref.[8,9]). Let $E_{1}=$ $\{e, w, b\} \subset E_{X}$ and $f_{1}, f_{2} \in\left([0,1]^{X}\right)^{E_{1}}$ as follows:

$$
\begin{aligned}
& \left(f_{1}\right)_{e}=(0.8,0.1,0.5,0.9,0.6),\left(f_{1}\right)_{b}=(0.7,0.9,0.4,0.5,0.7) \\
& \left(f_{1}\right)_{w}=(0.4,0.7,0.5,0.6,0.5),\left(f_{2}\right)_{e}=(0.5,0.9,0.4,0.8,0.4) \\
& \left(f_{2}\right)_{b}=(0.3,1.0,0.2,0.4,0.5),\left(f_{2}\right)_{w}=(0.5,0.4,0.8,0.5,0.1)
\end{aligned}
$$

Then we obtain:

$$
\begin{aligned}
& \left(f_{1} \vee f_{2}\right)_{e}=(0.8,0.9,0.5,0.9,0.6),\left(f_{1} \vee f_{2}\right)_{b}=(0.7,1.0,0.4,0.5,0.7) \\
& \left(f_{1} \vee f_{2}\right)_{w}=(0.5,0.7,0.8,0.6,0.5),\left(f_{1} \wedge f_{2}\right)_{e}=(0.5,0.1,0.4,0.8,0.4) \\
& \left(f_{1} \wedge f_{2}\right)_{b}=(0.3,0.9,0.2,0.4,0.5),\left(f_{1} \wedge f_{2}\right)_{w}=(0.4,0.4,0.5,0.5,0.1)
\end{aligned}
$$

For $K=\left\{k_{1}, k_{2}\right\}$, we define a $[0,1]$-fuzzy $\left(K, E_{1}\right)$-soft topology $\mathcal{T}_{X}: K \rightarrow$ $[0,1]^{\left([0,1]^{X}\right)^{E_{1}}}$ as follows:

$$
\begin{aligned}
& \left(\mathcal{T}_{X}\right)_{k_{1}}(f)= \begin{cases}1, & \text { if } f=0_{X} \text { or } 1_{X}, \\
0.7, & \text { if } f=f_{1} \\
0.4, & \text { if } f=f_{2} \\
0.5, & \text { if } f=f_{1} \vee f_{2} \\
0.6, & \text { if } f=f_{1} \wedge f_{2} \\
0, & \text { otherwise. }\end{cases} \\
& \left(\mathcal{T}_{X}\right)_{k_{2}}(f)= \begin{cases}1, & \text { if } f=0_{X} \text { or } 1_{X}, \\
0.5, & \text { if } f=f_{1} \\
0, & \text { otherwise. }\end{cases}
\end{aligned}
$$


From Theorem $14(1)$, we obtain a $[0,1]$-fuzzy $\left(K, E_{1}\right)$-soft closure operator $\mathcal{C}_{\mathcal{T}_{X}}$ : $K \times\left([0,1]^{X}\right)^{E_{1}} \times(0,1] \rightarrow\left([0,1]^{X}\right)^{E_{1}}$ as follows:

$$
\begin{gathered}
\mathcal{C}_{\mathcal{T}_{X}}\left(k_{1}, f, r\right)= \begin{cases}1_{X}, & \text { if } f=1_{X}, r \in(0,1], \\
f_{1}^{*}, & \text { if } f \sqsubseteq f_{1}^{*}, f \nsubseteq f_{1}^{*} \wedge f_{2}^{*}, r \leq 0.7, \\
f_{2}^{*}, & \text { if } f \sqsubseteq f_{2}^{*}, f \nsubseteq f_{1} \wedge f_{2}^{*}, r \leq 0.4, \\
f_{1}^{*} \wedge f_{2}^{*} & \text { if } f \sqsubseteq f_{1}^{*} \wedge f_{2}^{*}, r \leq 0.5 \\
f_{1}^{*} \vee f_{2}^{*} & \text { if } f \sqsubseteq f_{1}^{*} \vee f_{2}^{*}, f \nsubseteq f_{1}^{*}, f \nsubseteq f_{2}^{*}, r \leq 0.6, \\
0_{X}, & \text { otherwise. }\end{cases} \\
\mathcal{C}_{\mathcal{T}_{X}}\left(k_{2}, f, r\right)= \begin{cases}1_{X}, & \text { if } f=1_{X}, r \in(0,1], \\
f_{1}^{*}, & \text { if } f \sqsubseteq f_{1}^{*}, r \leq 0.5, \\
0_{X}, & \text { otherwise. }\end{cases}
\end{gathered}
$$

(2) Let $Z=\left\{z_{1}, z_{2}, z_{3}, z_{4}\right\}$ and $E_{3}=\{p, q, r, s\}$ be given. Define $\chi_{\phi}$ : $\left(L^{X}\right)^{E_{3}} \rightarrow\left(L^{X}\right)^{E_{1}}$ as follows

$$
\begin{gathered}
\chi\left(z_{1}\right)=\chi\left(z_{2}\right)=h_{1}, \chi\left(z_{3}\right)=h_{4}, \chi\left(z_{4}\right)=h_{5} \\
\phi(p)=\phi(q)=e, \phi(r)=b, \phi(s)=w .
\end{gathered}
$$

Since $\chi_{\phi}^{-1}\left(f_{1}\right)(p)\left(z_{1}\right)=f_{1}(\phi(p))\left(\chi\left(z_{1}\right)\right)=0.8$, we have

$$
\begin{array}{ll}
\chi_{\phi}^{-1}\left(f_{1}\right)(p)=(0.8,0.8,0.9,0.6), & \chi_{\phi}^{-1}\left(f_{1}\right)(q)=(0.8,0.8,0.9,0.6) \\
\chi_{\phi}^{-1}\left(f_{1}\right)(r)=(0.7,0.7,0.5,0.7), & \chi_{\phi}^{-1}\left(f_{1}\right)(s)=(0.4,0.4,0.6,0.5) \\
\chi_{\phi}^{-1}\left(f_{2}\right)(p)=(0.5,0.5,0.8,0.4), & \chi_{\phi}^{-1}\left(f_{2}\right)(q)=(0.5,0.5,0.8,0.4) \\
\chi_{\phi}^{-1}\left(f_{2}\right)(r)=(0.3,0.3,0.4,0.5), & \chi_{\phi}^{-1}\left(f_{2}\right)(s)=(0.5,0.5,0.5,0.1) .
\end{array}
$$

From Theorem 13, we obtain a [0,1]-fuzzy $\left(K, E_{3}\right)$-soft topology $\mathcal{T}_{Z}: K \rightarrow$ $[0,1]^{\left([0,1]^{Z}\right)^{E_{3}}}$ as follows:

$$
\begin{aligned}
& \left(\mathcal{T}_{Z}\right)_{k_{1}}(h)= \begin{cases}1, & \text { if } h=0_{Z} \text { or } 1_{Z} \\
0.7, & \text { if } h=\chi_{\phi}^{-1}\left(f_{1}\right) \\
0.4, & \text { if } h=\chi_{\phi}^{-1}\left(f_{2}\right) \\
0.5, & \text { if } h=\chi_{\phi}^{-1}\left(f_{1}\right) \vee \chi_{\phi}^{-1}\left(f_{2}\right) \\
0.6, & \text { if } h=\chi_{\phi}^{-1}\left(f_{1}\right) \wedge \chi_{\phi}^{-1}\left(f_{2}\right) \\
0, & \text { otherwise. }\end{cases} \\
& \left(\mathcal{T}_{Z}\right)_{k_{2}}(h)= \begin{cases}1, & \text { if } h=0_{Z} \text { or } 1_{Z} \\
0.5, & \text { if } h=\chi_{\phi}^{-1}\left(f_{1}\right) \\
0, & \text { otherwise. }\end{cases}
\end{aligned}
$$


From Theorem 14, we obtain a $[0,1]$-fuzzy $\left(K, E_{3}\right)$-soft closure operator $\mathcal{C}_{\mathcal{T}_{Z}}: K \times\left([0,1]^{Z}\right)^{E_{3}} \times(0,1] \rightarrow\left([0,1]^{Z}\right)^{E_{3}}$ as follows:

$$
\mathcal{C}_{\mathcal{T}_{Z}}(h, r)= \begin{cases}1_{X}, & \text { if } h=1_{X}, r \in(0,1], \\ \chi_{\phi}^{-1}\left(f_{1}^{*}\right), & \text { if } h \sqsubseteq \chi_{\phi}^{-1}\left(f_{1}^{*}\right), h \nsubseteq \chi_{\phi}^{-1}\left(f_{1}^{*}\right) \wedge \chi_{\phi}^{-1}\left(f_{2}^{*}\right), r \leq 0.7 \\ \chi_{\phi}^{-1}\left(f_{2}^{*}\right), & \text { if } h \sqsubseteq \chi_{\phi}^{-1}\left(f_{2}^{*}\right), h \nsubseteq \chi_{\phi}^{-1}\left(f_{1}^{*}\right) \wedge \chi_{\phi}^{-1}\left(f_{2}^{*}\right), r \leq 0.4 \\ \chi_{\phi}^{-1}\left(f_{1}^{*}\right) \wedge \chi_{\phi}^{-1}\left(f_{2}^{*}\right), & \text { if } h \sqsubseteq \chi_{\phi}^{-1}\left(f_{1}^{*}\right) \wedge \chi_{\phi}^{-1}\left(f_{2}^{*}\right), r \leq 0.5 \\ \chi_{\phi}^{-1}\left(f_{1}^{*}\right) \vee \chi_{\phi}^{-1}\left(f_{2}^{*}\right), & \text { if } h \sqsubseteq \chi_{\phi}^{-1}\left(f_{1}^{*}\right) \vee \chi_{\phi}^{-1}\left(f_{2}^{*}\right) \\ & h \nsubseteq \chi_{\phi}^{-1}\left(f_{1}^{*}\right), h \nsubseteq \chi_{\phi}^{-1}\left(f_{2}^{*}\right), r \leq 0.6 \\ 0_{X}, & \text { otherwise. }\end{cases}
$$

Example 16. Let $X$ and $E_{X}$ be given as Example 3.3. Define a binary operation $\odot$ on $[0,1]$ by

$$
\begin{gathered}
x \odot y=\max \{0, x+y-1\}, x \rightarrow y=\min \{1-x+y, 1\} \\
x \oplus y=\min \{1, x+y\}, x^{*}=1-x .
\end{gathered}
$$

Then $\left([0,1], \odot, \oplus, \rightarrow{ }^{*}, 0,1\right)$ be a complete residuated lattice. Let $E=\{b, w, c\} \subset$ $E_{X}, f \in\left([0,1]^{X}\right)^{E}$ be a fuzzy soft set as follow:

$$
\begin{gathered}
f_{b}=(0.5,0.3,0.5,0.6,0.2) \quad f_{c}=(0.1,0.2,0.6,0.5,0.5) \\
f_{w}=(0.4,0.4,0.5,0.6,0.6) \\
(f \odot f)_{b}=(0.0,0.0,0.0,0.2,0.0), \quad(f \odot f)_{c}=(0.0,0.0,0.2,0.0,0.0) \\
(f \odot f)_{w}=(0.0,0.0,0.0,0.2,0.2) .
\end{gathered}
$$

(1) Let $K=\left\{k_{1}, k_{2}\right\}$ be given. Define a [0,1]-fuzzy $(K, E)$-soft topology $\mathcal{T}_{X}$ : $K \rightarrow[0,1]^{\left([0,1]^{X}\right)^{E}}$ as follows:

$$
\begin{aligned}
& \left(\mathcal{T}_{X}\right)_{k_{1}}(h)= \begin{cases}1, & \text { if } h=0_{X} \text { or } h=1_{X}, \\
0.6, & \text { if } h=f \\
0.3, & \text { if } h=f \odot f \\
0, & \text { otherwise. }\end{cases} \\
& \left(\mathcal{T}_{X}\right)_{k_{2}}(h)= \begin{cases}1, & \text { if } h=0_{X} \text { or } h=1_{X}, \\
0.4, & \text { if } h=f \\
0, & \text { otherwise. }\end{cases}
\end{aligned}
$$


We obtain a $[0,1]$-fuzzy $(K, E)$-soft closure operator $\mathcal{C}_{\mathcal{T}_{X}}: K \times\left([0,1]^{X}\right)^{E} \times$ $(0,1] \rightarrow\left([0,1]^{X}\right)^{E}$ as follows:

$$
\begin{aligned}
& \mathcal{C}_{\mathcal{T}_{X}}\left(k_{1}, h, r\right)= \begin{cases}1_{X}, & \text { if } h=1_{X}, r \in(0,1], \\
f^{*}, & \text { if } h \sqsubseteq f^{*}, r \leq 0.6 \\
f^{*} \oplus f^{*} & \text { if } h \sqsubseteq f^{*} \oplus f^{*}, h \nsubseteq f^{*}, r \leq 0.3 \\
0_{X}, & \text { otherwise. }\end{cases} \\
& \mathcal{C}_{\mathcal{T}_{X}}\left(k_{2}, h, r\right)= \begin{cases}1_{X}, & \text { if } h=1_{X}, r \in(0,1], \\
f^{*}, & \text { if } h \sqsubseteq f^{*}, r \leq 0.4 \\
0_{X}, & \text { otherwise. }\end{cases}
\end{aligned}
$$

(2) Let $Z=\left\{z_{1}, z_{2}, z_{3}, z_{4}\right\}$ and $E_{4}=\{p, q, r\}$ be given. Define $\rho_{\psi}$ : $\left([0,1]^{Z}\right)^{E_{4}} \rightarrow\left([0,1]^{Z}\right)^{E}$ as follows

$$
\begin{aligned}
& \rho\left(z_{1}\right)=h_{2}, \rho\left(z_{2}\right)=h_{1}, \rho\left(z_{3}\right)=h_{5}, \rho\left(z_{4}\right)=h_{3} \\
& \psi(p)=c, \psi(q)=w, \psi(r)=b . \\
& \rho_{\psi}^{-1}(f) \quad z_{1} \quad z_{2} \quad z_{3} \quad z_{4} \\
& \begin{array}{lllll}
p & 0.2 & 0.1 & 0.5 & 0.6
\end{array} \\
& \begin{array}{lllll}
q & 0.4 & 0.4 & 0.6 & 0.5
\end{array} \\
& \begin{array}{lllll}
r & 0.3 & 0.5 & 0.2 & 0.5
\end{array} \\
& \rho_{\psi}^{-1}(f \odot f) \quad z_{1} \quad z_{2} \quad z_{3} \quad z_{4} \\
& \begin{array}{lllll}
p & 0.0 & 0.0 & 0.0 & 0.2
\end{array} \\
& \begin{array}{lllll}
q & 0.0 & 0.0 & 0.2 & 0.0
\end{array} \\
& \begin{array}{lllll}
r & 0.0 & 0.0 & 0.0 & 0.0
\end{array} \\
& \rho_{\psi}^{-1}\left(f^{*} \oplus f^{*}\right) \quad z_{1} \quad z_{2} \quad z_{3} \quad z_{4} \\
& \begin{array}{lllll}
p & 1.0 & 1.0 & 1.0 & 0.8
\end{array} \\
& \begin{array}{lllll}
q & 1.0 & 1.0 & 0.8 & 1.0
\end{array} \\
& \begin{array}{lllll}
r & 1.0 & 1.0 & 1.0 & 1.0
\end{array}
\end{aligned}
$$

From Theorem 13, we obtain a [0,1]-fuzzy $\left(K, E_{4}\right)$-soft topology $\mathcal{T}_{Z}: K \rightarrow$

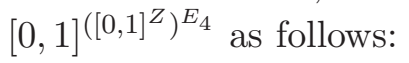

$$
\left(\mathcal{T}_{Z}\right)_{k_{1}}(h)= \begin{cases}1, & \text { if } h=0_{Z} \text { or } 1_{Z} \\ 0.6, & \text { if } h=\varphi_{\psi}^{-1}(f) \\ 0.3, & \text { if } h=\varphi_{\psi}^{-1}(f) \odot \varphi_{\psi}^{-1}(f) \\ 0, & \text { otherwise. }\end{cases}
$$




$$
\left(\mathcal{T}_{Z}\right)_{k_{2}}(h)= \begin{cases}1, & \text { if } h=0_{Z} \text { or } 1_{Z} \\ 0.4, & \text { if } h=\varphi_{\psi}^{-1}(f) \\ 0, & \text { otherwise. }\end{cases}
$$

From Theorem 14, we obtain a $[0,1]$-fuzzy $\left(K, E_{4}\right)$-soft closure operator $\mathcal{C}_{\mathcal{T}_{Z}}$ : $K \times\left([0,1]^{Z}\right)^{E_{4}} \times(0,1] \rightarrow\left([0,1]^{Z}\right)^{E_{4}}$ as follows:

$$
\begin{gathered}
\mathcal{C}_{\mathcal{T}_{Z}}\left(k_{1}, h, r\right)= \begin{cases}1_{Z}, & \text { if } h=1_{Z}, r \in(0,1], \\
\rho_{\psi}^{-1}\left(f^{*}\right), & \text { if } h \sqsubseteq \rho_{\psi}^{-1}\left(f^{*}\right), ; r \leq 0.6 \\
\rho_{\psi}^{-1}\left(f^{*}\right) \oplus \rho_{\psi}^{-1}\left(f^{*}\right) & \text { if } h \sqsubseteq \rho_{\psi}^{-1}\left(f^{*} \oplus f^{*}\right) \\
0_{Z}, & h \nsubseteq \rho_{\psi}^{-1}\left(f^{*}\right), r \leq 0.3, \\
& \text { otherwise. }\end{cases} \\
\mathcal{C}_{\mathcal{T}_{Z}}\left(k_{2}, h, r\right)= \begin{cases}1_{Z}, & \text { if } h=1_{Z}, r \in(0,1], \\
\rho_{\psi}^{-1}\left(f^{*}\right), & \text { if } h \sqsubseteq \rho_{\psi}^{-1}\left(f^{*}\right), r \leq 0.4 \\
0_{Z}, & \text { otherwise. }\end{cases}
\end{gathered}
$$

\section{Acknowledgments}

This work was supported by Jeju National University (2016)

\section{References}

[1] A. Aygünoglu, H. Aygün, Introduction to fuzzy soft groups, Computers and Mathematics with Appl., 58 (2009), 1279-1286.

[2] A. Aygünoglu, V. Cetkin, H. Aygün, An introduction to fuzzy soft topological spaces, Hacettepe Journal of Math. and Stat., 43 (2)(2014), 193-204

[3] V. Cetkin, Alexander P. S̆ostak, H. Aygün, An Approach to the Concept of Soft fuzzy Proximity, Abstract and Applied Analysis Stat. (2014), doi: 10.1155/2014/782583.

[4] V. Cetkin, H. Aygün, On fuzzy Soft topogenous structure, Journal of Intelligent and Fuzzy Systems, 27(1) (2014), 247-255.

[5] V. Cetkin, H. Aygün, On soft fuzzy closure and interior operator, Utilitas Mathematics, In press.

[6] D. Čimoka, A.Šostak, L-fuzzy syntopogenous structures, Part I: Fundamentals and application to $L$-fuzzy topologies, $L$-fuzzy proximities and $L$-fuzzy uniformities, Fuzzy Sets and Systems, 232 (2013), 74-97.

[7] F. Feng, X. Liu, V.L. Fotea, Y.B. Jun, Soft sets and soft rough sets, Information Sciences, 181 (2011), 1125-1137, doi: 10.1016/j.ins.2010.11.004. 
[8] P. Hájek, Metamathematices of Fuzzy Logic, Kluwer Academic Publishers, Dordrecht, 1998, doi: 10.1007/978-94-011-5300-3.

[9] U. Höhle, S.E. Rodabaugh, Mathematics of Fuzzy Sets: Logic, Topology, and Measure Theory, The Handbooks of Fuzzy Sets Series 3, Kluwer Academic Publishers, Boston, 1999, doi: 10.1007/978-1-4615-5079-2.

[10] Y.C.Kim, A.A. Ramadan, $L$-fuzzy $(K, E)$-soft topologies and $L$-fuzzy $(K, E)$-soft closure operators, Int. J. of Pure and Applied Math. 107 (4)(2016), 1073-1088, doi: 10.12732/ijpam.v107i4.24.

[11] P.K. Maji, R. Biswas, A.R. Roy, Fuzzy soft sets, Journal of Fuzzy Mathematics, 9 (3) (2001), 589-602.

[12] P.K. Maji, R. Biswas, A.R. Roy, Soft set theory, Computers Mathematics with Appl., 45 (2003), 555-562.

[13] P. Majumdar, S.K. Samanta, Generalized fuzzy soft sets, Computers Mathematics with Appl., 59(2010), 1425-1432.

[14] D. Molodtsov, Soft set theory, Computers Mathematics with Appl., 37 (1999), 19-31.

[15] D. Pei, D. Miao, From soft sets to information systems, Granular Computing, 2005 IEEE, International Conferences on (2)(2005), 617-621.

[16] A.A. Ramadan, E.H.Elkordy, Yong Chan Kim, Relationships between $L$-fuzzy quasiuniform stuctures and L-fuzzy topologies, Journal of Intelligent and Fuzzy Systems, 28 (2015), 2319-2327.

[17] S.E. Rodabaugh, E.P. Klement, Topological and Algebraic Structures In Fuzzy Sets, The Handbook of Recent Developments in the Mathematics of Fuzzy Sets, Kluwer Academic Publishers, Boston, Dordrecht, London, 2003.

[18] A.R. Roy, P.K. Maji, A fuzzy soft set theoretic approach to decision making problems, International of Computational and Applied Mathematics, 203(2007), 412-418.

[19] M. Shabir, M. Naz, On soft topological spaces, Computers and Mathematics with Appl., 61 (2011), 1786-1799.

[20] B. Tanay, M.B. Kandemir, Topological structures of fuzzy soft sets, Computers and Mathematics with Appl., 61 (2011), 412-418, doi: 10.1016/j.camwa.2011.03.056. 
